\title{
Histologic analysis of temporomandibular joint adaptation to protrusive function in young adult rhesus monkeys (Macaca mulatta)
}

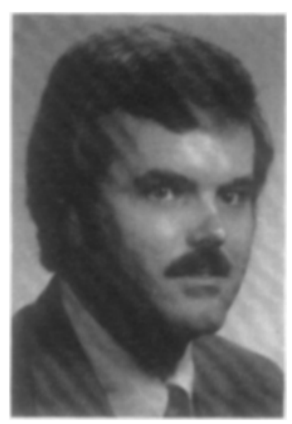

Dr. McNamara

\begin{abstract}
James A. McNamara, Jr., D.D.S., Ph.D., Robert J. Hinton, Ph.D., and Donald L. Hotiman, Ph.D., D.D.S.
Ann Arbor, Mich.

It is generally believed that the adult temporomandibular joint is incapable of a significant adaptive response to forces produced by functional jaw orthopedics. To evaluate this view, twelve young adult female rhesus monkeys were fitted with functional protrusive appliances for periods ranging from 2 to 24 weeks. Histologic analysis of the temporomandibular joint demonstrated that six of the experimental animals exhibited a tissue response that was qualitatively similar to that noted in juvenile animals. However, the response in young adults was greatly reduced in magnitude from that seen in juveniles and was not related to duration of treatment. It must also be noted that three adult animals developed cross-bites in response to appliance placement, and three animals who functioned anteriorly exhibited little or no detectable condylar response. This study indicates that while some adaptive capability may still be present in the temporomandibular joints of young adults, the potential magnitude of the joint response is limited and highly variable in occurrence.
\end{abstract}

Key words: Temporomandibular joint, rhesus monkey, functional protrusive appliance, jaw function, adult

The he past two decades have witnessed a growing number of studies seeking to investigate the adaptive potential of the craniofacial skeleton in response to experimental alterations designed to simulate clinical treatment. A considerable portion of this research effort has been directed toward an understanding of the growth and adaptation of the temporomandibular joint (TMJ). Yet, despite this fact, no consensus exists regarding the adaptability of the temporomandibular joint in response to alteration of its structural or functional environment. A primary reason for this lack of agreement arises from the apparent dependence of adaptive potential on the age or developmental stage of the animal. Thus, there is ample evidence that compensatory growth occurs at the temporomandibular joint, and especially the mandibular condyle, in response to altered occlusal function in young, growing animals. ${ }^{1-12}$ In particular, studies performed in our laboratory on monkeys and by Petrovic and associates on rats have shown that the condylar cartilage and bone in

From the Department of Anatomy, Center for Human Growth and Development (J. A. M. and R. J. H.), and School of Dentistry (D. L. H.), University of Michigan.

This study was supported in part by National Institutes of Health Grant DE 03610 growing animals respond to the altered neuromuscular function induced by a protrusive appliance by increased chondrocytic proliferation and subsequent bone deposition in a posterior and posterosuperior direction so as to reposition the condyle back within the mandibular fossa. Quantitative histologic studies have clarified the time-dependent nature of the adaptive response, indicating that the initial large changes in cartilaginous proliferation are progressively diminished when restoration of functional equilibrium is obtained. ${ }^{11}$ Moreover, Petrovic and co-workers ${ }^{5}$ have shown that repeated application of a postural hyperpropulsor during the growth period in rats results in a significant increase in over-all length of the mandible in treated animals relative to controls after both have reached maturity.

In contrast, the temporomandibular joint region in adult animals has been regarded as largely unresponsive to occlusal perturbations, ${ }^{8,10,13-16}$ with pathologic changes reported on occasion. ${ }^{17}$ These studies have emphasized the primacy of accommodative changes in the dentoalveolar region in the adaptive response of mature monkeys, prompting the observation by Ramfjord and $\mathrm{Ash}^{18}$ that there is a "need for adapting the occlusion to the joints rather than hoping for the joints to adapt to the occlusion, at least when considering the adult temporomandibular joint." Nevertheless, no 
large-scale studies comparable to those conducted on growing animals, especially involving quantification and/or detailed histologic analysis, have been performed on adult animals. In addition, it has become apparent that sex and degree of maturity of the "adult" animals, factors not always considered in previous studies, may be crucial to the results obtained. In fact, a recent study ${ }^{19}$ using mature rats suggests that the adaptive potential at the adult temporomandibular joint may not be as dormant as supposed. (See also Folke and Stallard $^{20}$ and Ehrlich et al. ${ }^{21}$ for similar indications.) Certainly the persistence of a cartilage layer in the condyle of adolescent and young adult monkeys and human beings has been well documented ., 22-25 In recognition of the increasing inclination of human beings in these age categories to seek orthodontic therapy ${ }^{26}$ and of some clinicians to attempt to provide functional jaw orthopedic treatment on an empirical basis, the present study was undertaken to analyze adaptive capabilities in the temporomandibular joint region of adult monkeys following an alteration in the mandibular postural position. The experimental design is similar to that employed in our earlier studies of growing (juvenile) monkeys. ${ }^{8-12}$ A major objective of this study is to determine if the adaptive changes observed in younger animals can be induced in mature animals who have nearly or completely ceased active growth.

\section{MATERIALS AND METHODS}

Nineteen adult female rhesus monkeys (Macaca mulatta) - seven control and twelve experimental animals-were used in this study. Although their exact ages were not known, all animals were physiologically mature with third molars in occlusion, making them at least $5 \frac{1 / 2}{2}$ to 6 years of age. ${ }^{27}$ These monkeys may correspond to human beings in their late teens to midtwenties. All animals were fed a normal Purina Monkey Chow diet.

For all experimental procedures, the animals were sedated with Ketamine $\mathrm{HCl}$ ( 7 to $15 \mathrm{mg}$. per kilogram intramuscularly) and Rompun (Xylazine) (1 to $2 \mathrm{mg}$. per kilogram intramuscularly). From mandibular and maxillary alginate impressions taken on each animal, a mandibular appliance was constructed so as to produce a protrusive centric occlusal relationship (Fig. 1). (See McNamara and Carlson ${ }^{11}$ for details.) The appliance resulted in a vertical (inferior) displacement of 2 to 3 $\mathrm{mm}$. and a horizontal (anterior) displacement of $5 \mathrm{~mm}$. Three animals developed a cross-bite in response to the appliance, with the result that little or no protrusion of the lower jaw was effected. The experimental (protrusive appliance) animals were studied for periods of 2 , $4,6,8,12$, and 24 weeks. Prior to sacrifice, a radio-

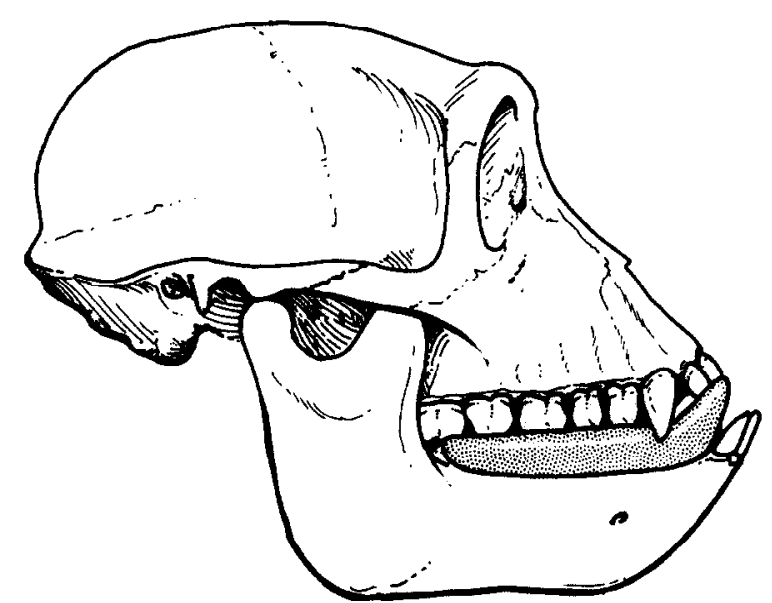

Fig. 1. Schematic representation of the protrusive appliance cemented on the mandibular arch. Note the forward and slight downward positioning of the mandible.

graph was obtained and the animal was given an intravenous injection of heparin (300 to 500 units per kilogram) to facilitate tissue preparation for histologic sectioning and staining. The animal was then anesthetized with Sernylan (phencyclidine $\mathrm{HCl}, 1$ to $2 \mathrm{mg}$. per kilogram intramuscularly) and sodium pentobarbital ( 8 to $10 \mathrm{mg}$. per kilogram intravenously), sacrificed by catheterization of the left ventricle or the carotid artery, and perfused with an AFA fixative (acetic acid, formalin, 95 percent ETOH).

After sacrifice, the head was placed into 10 percent NBF (neutral buffered formalin) prior to removal of the TMJ. The left and right temporomandibular joints were removed, immersed in Bouin's fixative for 72 hours, and then decalcified in Warshawsky's EDTA (ethylenediamine tetraacetic acid). Decalcification was usually complete in 3 to 6 weeks and was verified radiographically. Tissue preparation followed our routine laboratory dehydration and infiltration protocol. The tissues were embedded in paraffin, sectioned at 18 to 22 $\mu \mathrm{m}$, and stained with either hematoxylin and eosin or Masson's trichrome stain.

\section{RESULTS}

Temporomandibular joint microanatomy in the control and expcrimental samples was compared from both a qualitative and a quantitative standpoint.

\section{Qualitative analysis}

Control sample. Although there was some variabil ity in histologic appearance (Fig. 2), all of the control animals could be characterized by a common group of morphologic features. (See Zimmermann ${ }^{22}$ for similar observations.) In particular, although remnants of a 


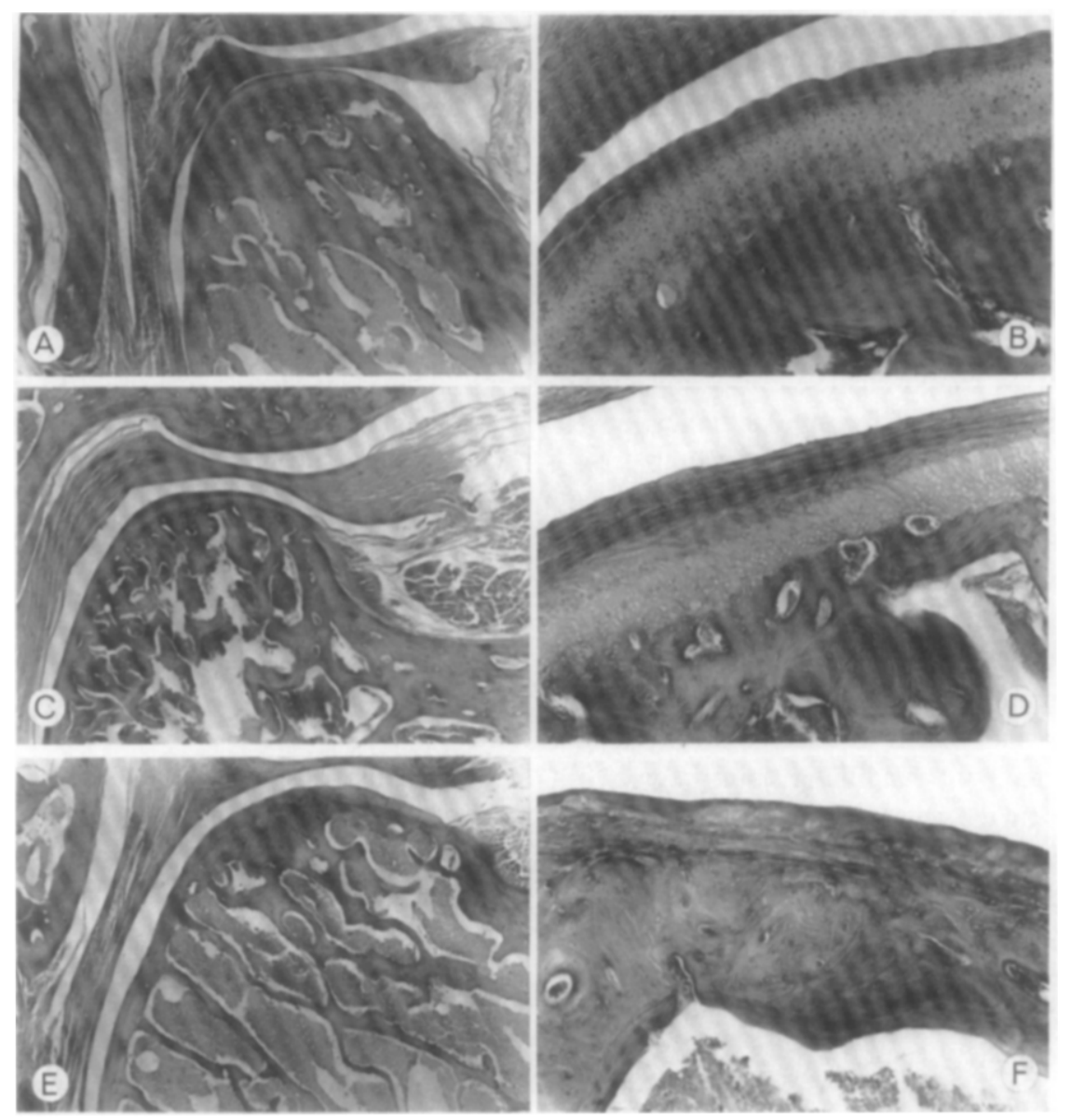

Fig. 2. Variation in condylar morphology in the control animals. A and B, Maximum cartilage proliferation. $\mathbf{C}$ and $\mathbf{D}$, Moderate cartilage proliferation. $\mathbf{E}$ and $\mathbf{F}$, Minimal cartilage proliferation. (Hematoxylin and eosin stain. Magnifications: A, C, E, × 12; B, D, F, $\times 40$. Sagittal section.)

cartilage layer could be distinguished in nearly all animals, the histologic appearance of this layer differed dramatically from that seen in animals at younger developmental stages. ${ }^{11,}{ }^{28} \mathrm{~A}$ marked reduction in the number of chondrocytes was observed in the adults as compared to juveniles or adolescents, and the presence of hypertrophic chondrocytes was rare. In addition, in a number of animals collagen bundles were faintly visible in the matrix, giving the tissue the appearance of fibrocartilage in many regions (Fig. $2, D$ ). In nearly all instances, a proliferative (prechondroblastic) zone between the articular tissue and the chondroblastic zone could not be reliably identified. It should be noted, however, that even though there were similarities in the morphologic features of the adult control condyles, there were also some striking differences, particularly in the relative amount of condylar cartilage present. Abundant cartilage was present in some condyles (Fig. 2, $A$ and $B$ ) while nearly absent in others (Fig. 2, $E$ and $F$ ).
A second major feature common to all control animals was the closing-off of the cartilage layer from the underlying medullary spaces by a coalescence of bony trabeculae and, in a number of cases, by a compact layer of remodeled bone (Fig. 2). This resulted in a relatively even, unbroken line of demarcation between the remaining cartilage and the bony "cap," with very few invaginations at the cartilage-bone interface. By comparison, such invaginations are the norm in rapidly growing animals at earlier developmental stages where they represent the sites of degeneration of hypertrophic chondrocytes and the deposition of bone on the remnants of calcified cartilage. ${ }^{29}$

Experimental sample. Response in the temporomandibular joint region of the experimental animals was varied, in part because of the development of cross-bites in three animals (with a resultant lack of functional protrusion) and perhaps interindividual variation. Of the remaining nine animals, three exhibited almost no perceivable response to the appliance 


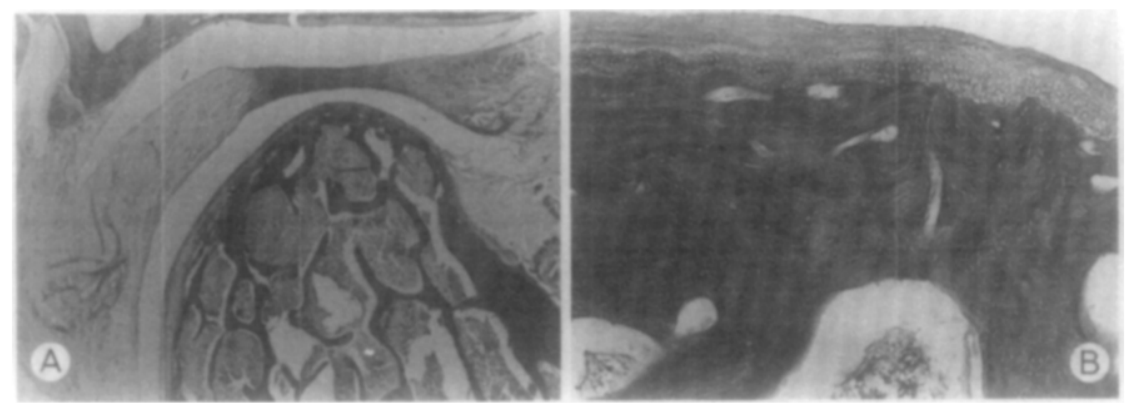

Fig. 3. A, Temporomandlbular jolnt region in a 12-week animal. Note lack of condylar cartilage hypertrophy. B, The appearance of the condylar cartilage in the same animal. Note the thickness of the bony cap and the limited number of cells present. (Magnifications: $A, \times 12 ; B, \times 40$.)

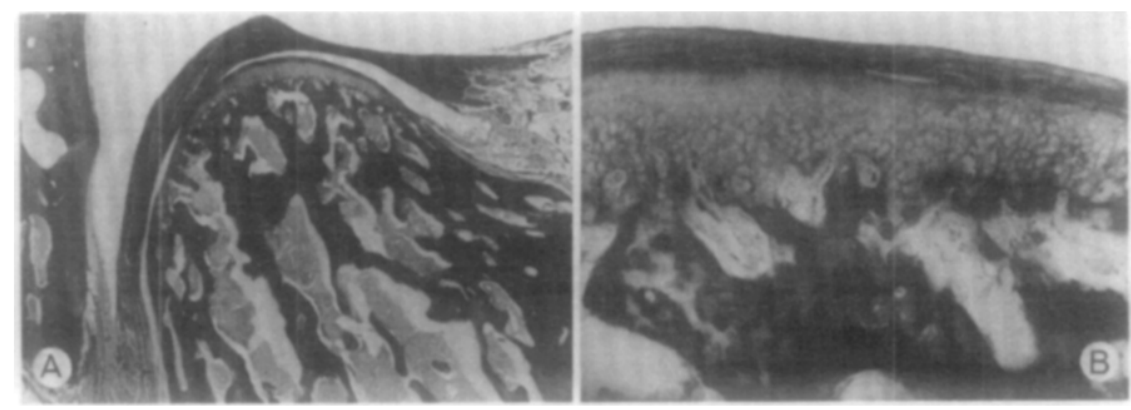

Fig. 4. A, Temporomandibular joint region of a 2-week animal. B, Appearance of condylar cartilage along the superior surface of the condyle. (Magnifications: A, $\times 12 ; \mathbf{B}, \times 40$.)
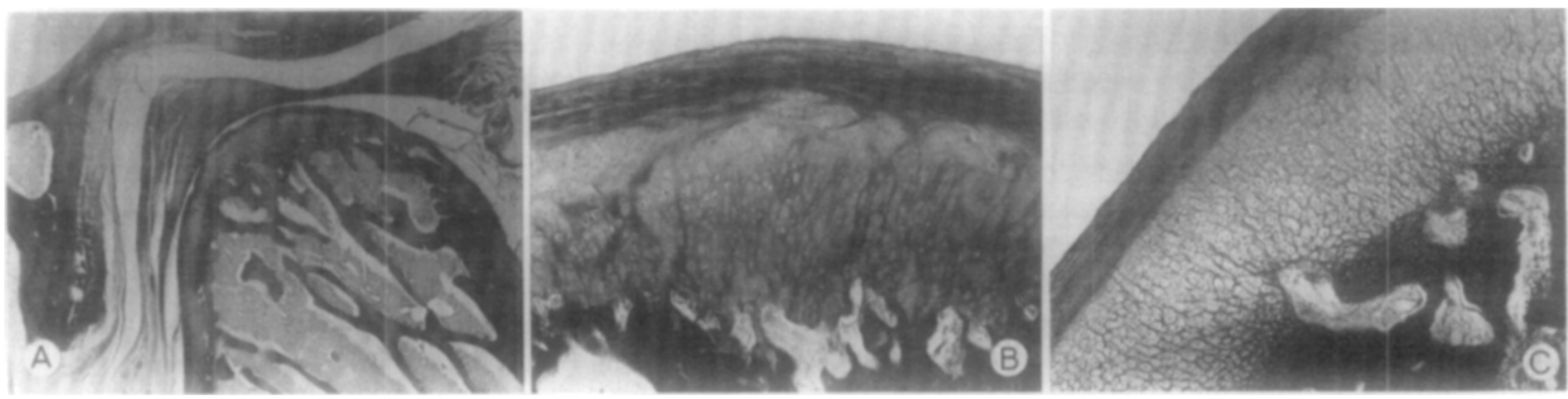

Fig. 5. A, The temporomandibular joint region of an 8-week experimental animal. Note the increased thickness of the condylar cartilage along the superior and posterior aspects of the condyle. Note also the bone deposition along the anterior border of the postglenoid spine. B, Proliferation of cartilage along the superior border of the condyle. $\mathbf{C}$, Proliferation of cartilage along the posterosuperior border of the condyle. (Magnifications: A, $\times 12$; B and C, $\times 40$.)

(Fig. 3). Except for some indications of slight chondrocytic proliferation, temporomandibular joint morphology resembled that of controls.

In the other animals, considerable hyperplasia of the prechondroblastic-chrondroblastic cartilage layer occurred, particularly in the superior and posterosuperior regions. In three of the four animals sacrificed after 2 and 4 weeks, this hypercellularity was manifest as a three-layer structure characteristic of sites of active growth in younger animals: a proliferative (prechondroblastic) layer giving way to a chondroblastic layer, with hypertrophic chondrocytes in the deeper layers (Fig. 4, $A$ ). Also, chondrocytic proliferation in these animals was accompanied in varying degrees by a highly invaginated cartilage-bone interface, with evidence of incorporation of calcified cartilage into newly formed bony spicules (Fig. 4, B). The sole exception to these statements among the 2-and 4-week animals was 


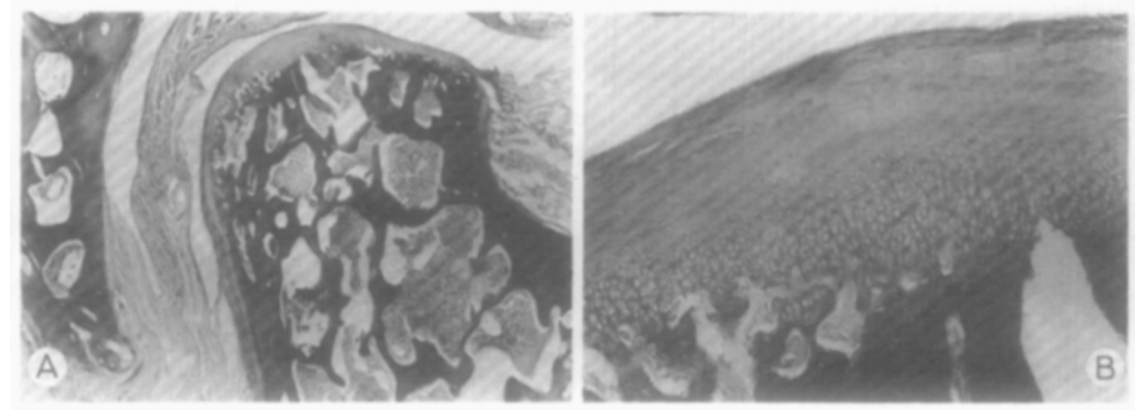

Fig. 6. A, Temporomandibular joint region of a 12-week experimental animal. Note the proliferation of cartilage superiorly and posterosuperiorly. B, Proliferation of cartilage along the posterosuperior aspect of the condyle. (Magnifications: A, $\times 12 ; \mathrm{B}, \times 40$.)

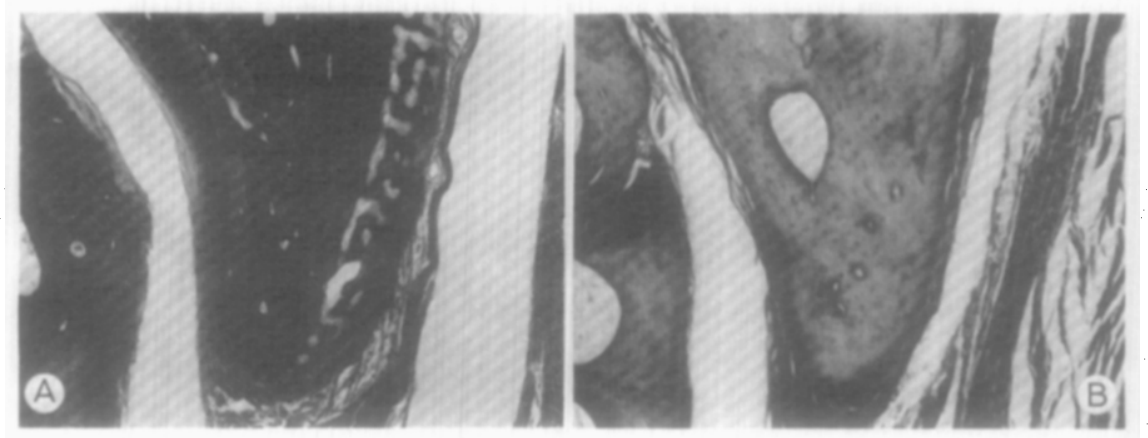

Fig. 7. A, Proliferation of new bone along the anterior surface of the postglenoid spine in an 8-week animal. B, Postglenoid spine in a control animal. (Magnifications: $A, \times 12 ; B, \times 24$.)

the 2-week animal which developed a cross-bite; this animal strongly resembled the control animals (albeit with perhaps greater cellularity), exhibiting no invagination of the bony cortical plate and little or no cartilage hypertrophy. The animals in the remaining groups $(6,8,12$, and 24 weeks) showed qualitatively similar histomorphology, including moderate to pronounced hypercellularity of the chondrocytic layers coupled with regions of invagination of the bony cortical plate exhibiting signs of active growth. In most animals, this area of apparent growth was located in the superior or posterosuperior region of the condylar cartilage, and only occasionally at its posterior aspect. (Fig. 5 depicts a typical example in one of the 8-week animals.) It is worthy of note that three of the four animals in the 12and 24-week groups (Fig. 6) showed evidence of continuing, active growth, with cartilage cellularity that appeared to exceed that in any control animal (Fig. 2). Beginning with the animals sacrificed at 8 weeks, adaptations were also noted in the microanatomy of the temporal joint component. Specifically, evidence of bone deposition on the anterior surface of the postglenoid spine and posterior surface of the mandibular fossa was present in both 8-week animals (Fig. 5, $A$ and
Fig. 7), one of two 12-week animals, and one of two 24-week animals.

As noted earlier, the responses outlined above were not observed in every experimental animal, nor to the same extent in every animal. For example, one of the 6week animals was virtually indistinguishable histologically from the animals in the control sample, while the other 6-week animal developed a severe cross-bite and showed few differences in the condylar cartilage from controls. In addition, two of the other experimental animals showed a differential proliferation of chondrocytes in various regions along the sagittal profile of the condyle but displayed little or no break in the cortical plate at the cartilage-bone interface. However, the majority of experimental subjects (excluding those in which functional protrusion did not occur because of a cross-bite) manifested a consistent histologic pattern which was qualitatively distinct from that seen in controls.

Cross-bite animals. Temporomandibular joint adaptations in those animals that developed a cross-bite instead of a functional protrusion of the lower jaw were of interest in their own right. The cross-bite animal sacrificed at 4 weeks exhibited hypercellularity of the 


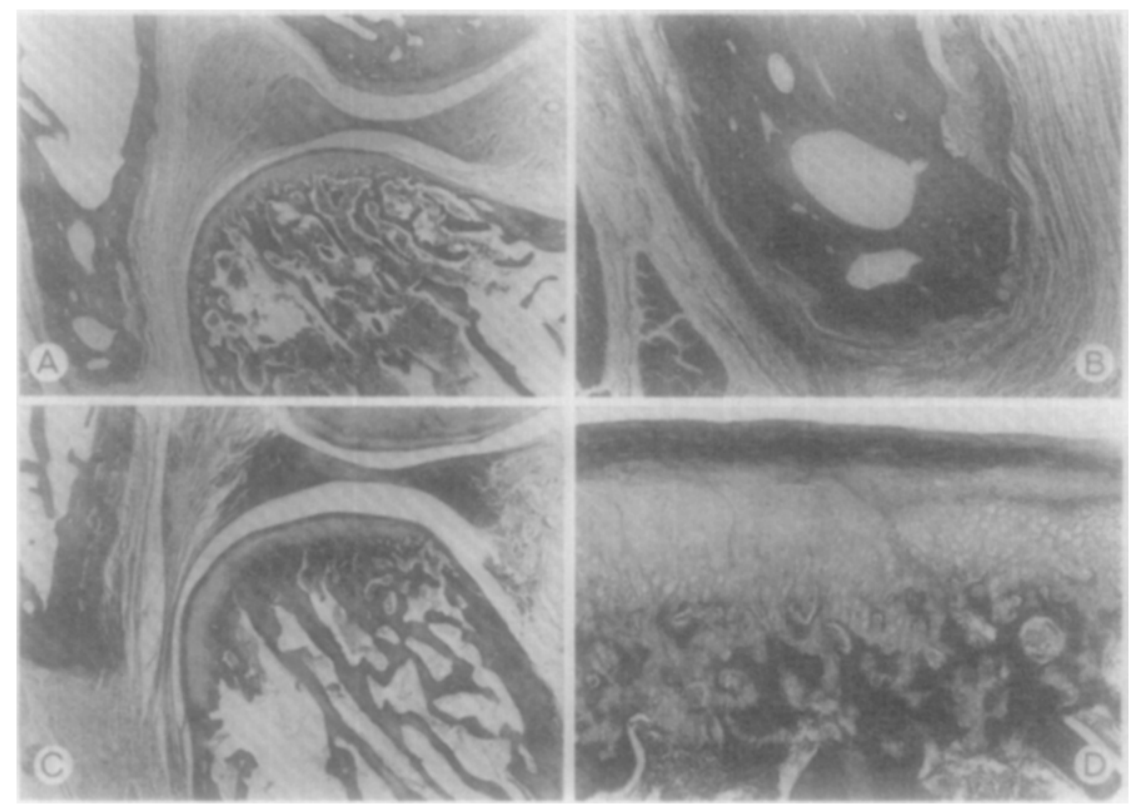

Fig. 8. Condylar adaptation in a 4-week cross-bite animal in which the mandible deviated to the right. A, Right temporomandibular joint region with resorption of bone along the anterior border of the postglenoid spine. Note the lack of cartilage proliferation along the posterior border of the mandible. $B$, Resorption of bone along the anterior border of the right postglenoid spine. C, Left temporomandibular joint region in same animal. Note the deposition of new bone along the postglenoid spine and the proliferation of the condylar cartilage. D, Proliferation of condylar cartilage along the superior surface of the left condyle. (Magnifications: A and C, $\times 12$; B and D, $\times 40$.)

chondroblastic layer together with a highly invaginated cartilage-bone interface in both joints.* The right temporomandibular joint, which had cartilage thicknesses from 14 to 110 percent greater than those on the left side at comparable locations, displayed distinct evidence of new bone formation on the anterior aspect of the postglenoid spine, while the left postglenoid spine contained foci of resorption along its anterior border (Fig. 8). The 6-week cross-bite animal showed little difference in condylar morphology from that seen in controls, but there were distinct areas of resorption on the anterior surface of the postglenoid spine in both joints. The 2-week animal with a cross-bite presented no notable differences from controls; in light of the tissue responses noted in cross-bite animals at later stages, it is possible that the lack of response in the 2-week animal may be due to the abbreviated time interval.

*Although a cycling female, this animal was perhaps a little less maturc than the others. While the mandibular third molar had reached the level of the occlusal plane, the maxillary third molar, although erupted, had not quite reached this level. Thus, the vitality of the condylar cartilage, rivaling that of any animal in either the control or experimental groups, may be due in part to its slight developmental immaturity.

\section{Quantitative analysis}

In order to test whether changes in the condylar cartilage display a diminished response over time as seen in juvenile animals, ${ }^{11}$ thickness of the prechondroblastic-chondroblastic cartilaginous layer was measured by means of a reticule inserted into the eyepiece of a Zeiss Photomicroscope III. The thickness of the cartilage layers was measured perpendicular to the articular surface at the posterior, posterosuperior, and superior regions along the circumference of the condyle. ${ }^{11}$ Linear regressions* were computed for each region of the condyle to determine the association between cartilage thickness and duration of treatment (Fig. 9, Table I). Although cartilage thickness appears to undergo a slight decrease with treatment duration, no significant relationship could be detected comparable to that found in juvenile animals. However, Fig. 9 substantiates the qualitative assessment that the adaptive response is more pronounced in the superior and posterosuperior regions of the condyle, with less response in the posterior region. Thickness of the condy-

*Regressions were performed for right side measurements, left side measurements, and both sides combined. Data from animals who developed cross-bites were excluded from the analyses. 

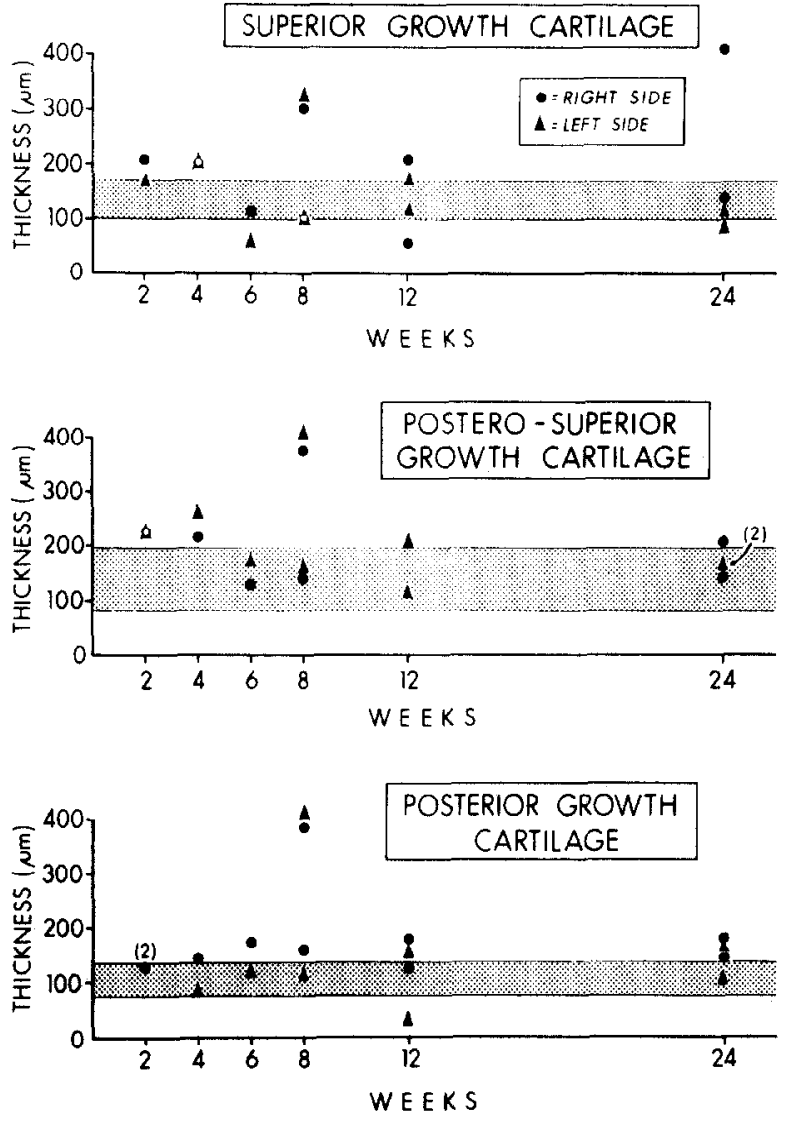

Flg. 9. Least squares regression analysis of adaptive responses in the thickness of condylar cartilage relative to duration of treatment. Data points for each experimental animal are indicated by side. Stippled area indicates the range of condylar thickness to the first standard deviation in the control animals.

lar cartilage was consistently greater in experimental animals relative to controls at all locations studied (Table I), although the differences were not significant, perhaps because of the large amount of variation in the experimental sample.

\section{DISCUSSION}

The results of this study provide evidence that in the majority of the experimental animals the condylar cartilage in young adult monkeys was affected by alteration of the biomechanical environment of the temporomandibular joint. Functional protrusion of the lower jaw resulted in an increase in the number of cells in the prechondroblastic-chondroblastic layer accompanied by a deposition of new bone at the cartilage-bone interface. Although not as pronounced, this response is qualitatively very similar to that documented in growing monkeys that were fitted with a similar protrusive appliance. While the magnitude of the response (as assessed by thickness of the prechondroblastic-chon- droblastic layer) decreased some what as the duration of treatment increased, the time-dependent nature of the adaptive response in adult animals was not nearly as marked as that seen in juvenile monkeys. (Regressions of adult cartilage thickness on treatment duration were not significant.) However, the density of chondroblasts in most experimental animals was consistently greater than that in the control sample, suggesting that a true change in condylar morphology had taken place in the experimental animals. Apart from the magnitude of the response, the other main difference observed between growing and young adult animals concerned the localization of the response along the circumference of the condyle. In the growing (juvenile) animals, cartilage proliferation was maximum in the postcrior, and to a lesser extent, the posterosuperior regions of the condyle. "In contrast, the most pronounced changes in the adult animals tended to occur primarily on the superior and posterosuperior aspects of the condyle, although there was perhaps more variability in location than in young animals. Assuming that the adaptive response is of the same sort as in juvenile animals (that is, a compensatory change to reposition the condyle back within the mandibular fossa), then these data would suggest that the adjustment in the adult condylar response was predominantly to the vertical displacement introduced by the appliance and only secondarily to the horizontal (protrusive) displacement. Such an assessment is admittedly subjective and demands replication and further study.

It is interesting that cross-bites were developed in one fourth of the experimental sample in response to appliance placement. Since cross-bites were never noted in juvenile animals fitted with appliances of similar construction, their occurrence in a substantial percentage of the adult animals is worthy of note. While the reasons for this situation are unclear, it may indicate a diminution in adults of the capability to habitually posture the lower jaw forward to the extent required by the appliance. Support for this inference derives from the fact that aberrant and even painful jaw postures, including constantly keeping the mouth open or penetration of the palate by the prongs of the appliance, were tolerated as apparent alternatives to protrusion of the lower jaw during occlusion. Whatever the case, tissue responses noted in the cross-bite animals may be explicable as adaptations induced by the asymmetric occlusion. In the 4-week cross-bite animal, the persistent distraction of one condyle from the mandibular fossa and the retrusion of the other condyle prompted by the nature of the cross-bite occlusion are consistent with the histologic findings: relatively greater cartilage thickness and bone deposition on the postglenoid spine 
Table I. Mean thickness (in microns) of the condylar cartilage in the control and experimental samples

\begin{tabular}{|c|c|c|c|c|c|c|c|c|c|c|c|c|}
\hline & \multicolumn{6}{|c|}{ Right } & \multicolumn{6}{|c|}{ Left } \\
\hline & \multicolumn{2}{|c|}{ Superior } & \multicolumn{2}{|c|}{$\begin{array}{c}\text { Posterosu- } \\
\text { perior }\end{array}$} & \multicolumn{2}{|c|}{ Posterior } & \multicolumn{2}{|c|}{ Superior } & \multicolumn{2}{|c|}{$\begin{array}{l}\text { Posterosu- } \\
\text { perior }\end{array}$} & \multicolumn{2}{|c|}{ Posterior } \\
\hline & Mean & $S . D$. & Mean & $S . D$. & Mean & $S . D$. & Mean & S.D. & Mean & S.D. & Mean & $S . D$. \\
\hline Control $(n=7)$ & 128.7 & 30.9 & 132.9 & 45.5 & 101.7 & 31.3 & 136.9 & 45.7 & 149.3 & 71.4 & 110.0 & 27.8 \\
\hline Experimental $(n=9)$ & 191.9 & 108.7 & 198.3 & 94.2 & 179.0 & 76.3 & 158.0 & 71.5 & 201.7 & 88.8 & 137.2 & 110.6 \\
\hline
\end{tabular}

Differences between means are not significant.

in one joint and the relatively reduced cartilage thickness (especially posteriorly) and postglenoid bone resorption at the contralateral joint. The resorption noted on the anterior surfaces of both postglenoid spines in the 6-week cross-bite animal may also be related to the presence of a cross-bite, since the animal seemed to adapt to the appliance by keeping its mouth open and shifting the lower jaw laterally to minimize the degree of opening. It has been suggested on various grounds ${ }^{22,28}$ that contact between the postglenoid spine and condyle may take place in monkeys, especially during gapes of some magnitude. In view of several studies linking habitual retropositioning of the condyle in the fossa with resorption on the anterior surface of the postglenoid spine, ${ }^{7}{ }^{30-32}$ the association of postglenoid remodeling changes with this animal's particular adaptation to the protrusive appliance does not seem unrcasonablc.

In the juvenile animal, it has been suggested that the proliferative chondrogenic response to altcred function is explicable in light of certain distinctive features of the condylar cartilage. Several workers ${ }^{29}, 33$ have noted that the articular layer is continuous with the outer fibrous periosteum of the neck of the condyle, while the prechondroblastic-chondroblastic layer corresponds to the inner osteogenic periosteal layer of the condylar neck. This fact has led to the assertion ${ }^{3,4}$ that the cells of the prechondroblastic zone are homologous with the preosteoblasts of the remainder of the periosteum of the mandible. On this basis, we have previously suggested that, just as mechanical forces may stimulate or inhibit periosteal osteogenesis they may act in similar fashion on the prechondroblasts of the condylar cartilage to produce the adaptive response. ${ }^{11}$ Studies employing autoradiography in growing animals have confirmed the primary role played by proliferation of prechondroblasts during normal growth of the condyle ${ }^{34-36}$ and in response to altered occlusal relationships. ${ }^{2}, 4,20,37$ However, there is little consensus concerning the potential for differentiation of prechondroblasts into chondroblasts in adult animals. Kanouse and associates ${ }^{36}$ have noted that the activity of cells at the cartilage-bone interface was much higher than the activity in the prechondroblastic zone in a young adult female monkey, in contrast to the greater activity in the prechondroblastic zone in younger animals. It seems reasonable that the chondrogenic response noted in this study involving adult animals may be the result of a reactivation of the cells of the prechondroblastic zone, with their subsequent differentiation into chondrocytes in deeper layers. This interpretation is supported by the work of Lindsay, ${ }^{19}$ who used autoradiographic techniques to study cellular activity in the condylar cartilage of mature rats fitted unilaterally with a bite-raising splint. He found a threefold increase in cellular proliferation on the splinted side, with higher labeling of cells in the prechondroblastic zone than in the hypertrophic zone. Using a similar experimental protocol on adult rats, Ehrlich and colleagues ${ }^{21}$ obscrved that occlusal changes introduced by the bite splint also preferentially altered the staining pattern for the cyclic nucleotides cAMP and cGMP in the prechondroblastic and hypertrophic zones. Staining for the cyclic nuclcotides, which have been postulated to act as mediators between mechanical stimuli and cellular proliferation, ${ }^{38}$ was greatly intensified in the prechondroblastic zone of treated animals but almost unaffected in the hypertrophic zone. In contrast, staining in control animals was uniform in both zones.

The implications of this study that a significant adaptive response may take place at the temporomandibular joint in some adult monkeys are in disagreement with the conclusions of several previous reports. Perhaps the best known of these are the series of studies performed by Ramfjord and co-workers. ${ }^{13}, 14,16$ They were unable to detect significant adaptive changes in the temporomandibular joints of adult monkeys subjected to functional protrusive and other appliances, and they attributed the adaptive response which did occur to compensatory changes in the dentoalveolar region. However, a number of factors make comparison of their results with those of the present study difficult. For instance, the control and experimental samples used in the various studies cited above were not 
always well matched for sex and age (to the extent that the latter can be determined in adult monkeys). Since young adult male monkeys continue to grow at a rate that is several times that seen in young adult females, ${ }^{39}$ our study included only young adult females in both control and experimental samples in an effort to minimize the amount of growth not associated with experimental intervention. Also, since third molars in females erupt, on the average, 4 to 8 months later than in males, ${ }^{27}$ the use of female animals with third molars in occlusion tended to bias the sample in favor of older animals, thereby making any results more conservative. In the Ramfjord studies, the experimental groups comprised either adult males of unknown age $\mathrm{e}^{13}$ or very old females. ${ }^{14}$ It may very well be the case that the potential for cellular response of the sort studied here is diminished in individuals considerably beyond young adulthood, a possibility which might be a mitigating circumstance in the lack of response noted by Ramfjord and associates. Second, the amount of functional protrusion introduced by the appliance was appreciably greater in our experiment $(5 \mathrm{~mm}$.) than in those of Ramfjord and associates $(1.5 \mathrm{~mm}$.). Conceivably, the greater occlusal imbalance created in the former instance could elicit an adaptive response of larger magnitude. Finally, previous studies of this problem have not, for the most part, undertaken detailed histologic analyses of the temporomandibular joint tissues. Howcver, it is interesting to note that Hiniker and Ramfjord, ${ }^{13}$ working with adult males, describe "some cartilage [covering] the bone on the articular surfaces" in a 2-week experimental animal and note that "these structures did not have a well-defined layer of cortical bone," characteristics which they attribute to the relatively young adulthood of the animal but which also resemble the morphology observed in 2-week experimental monkeys in this study. In addition, in the 6-week experimental animal they figure and describe a "narrow zone of newly-formed bone" on the distal aspect of the condylar neck as well as a thin layer of new bone on the anterior surface of the postglenoid spine and inferior surface of the mandibular fossa. Similarly Adams and co-authors ${ }^{7}$ comment that in the "youngest adult monkey"' exposed to Class II intermaxillary forces on one side and Class III on the other side (see also Meikle ${ }^{40}$ ), the condyle subjected to Class II force "demonstrated a more posteriorly directed endochondral ossification . . . compared to the control animals," and they liken this pattern to growth "to that seen in the younger [growing] experimental animals."

To varying degrees, the earlier studies cited above have concluded that condylar adaptive response in the adult temporomandibular joint, if it exists, is of such minimal magnitude as to be of no importance. The results of our study suggest that an adaptive response does indeed occur in most young adult animals, although its magnitude and even its presence may be considerably more variable than in growing animals. The question of magnitude of the response remains unresolved and may depend on a number of factors, including the age and sex of the individual, amount of condylar distraction produced by the appliance, and duration of treatment. For example, a recent cephalometric study of the effect of a bite-opening appliance on mandibular growth in adult female monkeys by two colleagues ${ }^{41}$ provides additional (quantitative) evidence of the potential for adaptive growth at the temporomandibular joint in young adults. In their study, distraction of the condyle from the mandibular fossa, brought about by the appliance, resulted in the deposition of an average of $1.5 \mathrm{~mm}$. of new bone along the posterosuperior aspect of the condyle in the experimental animals after 48 weeks. Thus, while dentoalveolar adaptations are undoubtedly of primary importance in adults, ${ }^{8}$ there are indications that compensatory changes at the temporomandibular joint may also contribute to the over-all accommodation to occlusal alterations, at lcast in young adults.

\section{Clinical implications}

The results of this study seem to indicate that in many instances the temporomandibular joint of the young adult animal is capable of some functional adaptation. This should not be surprising to the reader, even in light of previous studies which seemed to report contrary findings. The usefulness of this adaptive capability of the adult TMJ is perhaps best observed in patients following various orthognathic procedures of the mandible. ${ }^{42,43}$ In many of these cases, the mandibular condyle is repositioned or rotated to a moderate extent and TMJ pathosis is not a routine finding. By inference (and by radiographic indications of remodeling documented in such cases), this seems to indicate that some adaptive responses in the temporomandibular joint are elicited to accommodate to the new structural relationships within that region.

The relevance of this study to the use of functional jaw orthopedic appliances in adults must be stated with great caution. First, while in the majority of instances the qualitative response of the young adult condyle was similar in many respects to that observed in the juvenile animals, the magnitude of the response was greatly reduced in the adults. The amount of cartilage proliferation in the young adult animals was not nearly as great as observed in the young monkeys. Thus, it must be kept in mind that the biologically significant changes 
documented in this study may or may not be clinically significant.

The second reason for caution is that it must be noted that in our previous experiments with juvenile animals, condylar responses were observed in every animal studied from 2 to 12 weeks following appliance placement. ${ }^{11}$ In contrast, in three of the nine animals that functioned anteriorly (excluding the three additional cross-bite animals), the morphology of the condyles in the experimental animals could not be distinguished from controls. These nonresponding animals tended to have reduced cartilage thickness overlying a solid bony cortex similar to those control animals with a limited cartilage thickness without treatment (Fig. $2, E$ and $F)$. Since the exact age of the animals used was not known, it is not possible to directly evaluate whether the lack of response was primarily attributable to a more advanced age or to variation in responsiveness among individual animals. However, use of relative chronological ages obtained from assessment of occlusal and interproximal attrition indicates that the three nonresponding animals are all among the oldest in the experimental sample. ${ }^{44}$ Thus, it may be that as individuals age the condyle gradually loses the ability to respond functionally as the cartilage cells are lost. The problem facing the clinician is the prediction of which patients have sufficient capability to adapt to functional orthopedic treatment. A few millimeters of increased mandibular growth may be a reasonable goal in some patients. However, the correction of a mandibular deficiency that would otherwise require orthognathic surgery does not seem possible in the light of our experiments with adult monkeys.

\section{SUMMARY AND CONCLUSIONS}

The primary aim of this study has been to analyze histologic changes in the condylar cartilage of young adult monkeys (Macaca mulatta) in response to induced protrusive function. In the majority of animals in the experimental sample, a proliferative chondrogenic response accompanied by deposition of new bony trabeculae at the bone-cartilage interface was evident. These morphologic changes in the condylar cartilage resemble in form, although not in magnitude, the changes previously documented in growing monkeys undergoing a similar experimental protocol. In the growing monkeys, this chondrogenic response has been generally interpreted as an indication of the potential of the condylar cartilage to undergo compensatory growth as a means of re-establishing functional equilibrium. Although dentoalveolar adaptations are very likely the primary mechanism of occlusal adjustment in adults, the documentation of a qualitatively similar response in young adult monkeys suggests that the ability of the young adult condylar cartilage to participate in the over-all craniofacial adaptation to functional perturbations has been underestimated. Since all animals used in this study were physiologically mature adults with third molars in occlusion (thereby perhaps corresponding to human beings in their late teens to mid-twenties), the results reported here indicate that the assumption that the temporomandibular joints of young adults are stable and resistant to clinical intervention should be re-exarnined.

\section{REFERENCES}

1. Baume, L. J., and Derichsweiler, H.: Is the condylar growth center responsive to orthodontic therapy? An experimental study in Macaca mulatta, Oral Surg. 14: 347-362, 1961.

2. Charlier, J.-P., Petrovic, A., and Herrmann-Stutzmann, J.: Effects of mandibular hyperpropulsion on the prechondroblastic zone of young rat condyle, AM. J. ORTHOD. 55: 71-74, 1969.

3. Petrovic, A.: Mechanisms and regulation of mandibular condylar growth, Acta Morphol. Neerl-Scand. 10: 25-34, 1972.

4. Petrovic, A., Stutzmann, J. J., and Oudet, C. L.: Control processes in the postnatal growth of the condylar cartilage of the mandible. In McNamara, J. A., Jr. (editor): Determinants of mandibular form and growth, Monograph No. 4, Craniofacial Growth Series, Ann Arbor, 1975, Center for Human Growth and Development, The University of Michigan, pp. 101-154.

5. Petrovic A., Stutzmann, J., and Gasson, N.: The final length of the mandible: Is it genetically predetermined? In Carlson, D. S. (editor): Craniofacial biology, Monograph No. 10, Craniofacial Growth Series, Ann Arbor, 1981, Center for Human Growth and Development, The University of Michigan, pp. 105-126.

6. Stockli, P. W., and Willert, H. G.: Tissue reactions in the temporomandibular joint resulting from anterior displacement of the mandible in the monkey, AM. J. ORTHOD. 60: 142-155, 1971.

7. Adams, C. D., Meikle, M. C., Norwick, K. W., and Turpin, D. I.: Dentofacial remodeling produced by intermaxillary forces in Macaca mulatta, Arch. Oral Biol. 17: 1519-1535, 1972.

8. McNamara, J. A., Jr.: Neuromuscular and skeletal adaptations to altered orofacial function, Monograph No. 1, Craniofacial Growth Series, Ann Arbor, 1972, Center for Human Growth and Development, The University of Michigan.

9. McNamara, J. A., Jr.: Neuromuscular and skeletal adaptations to altered function in the orofacial region, AM. J. ORTHOD. 64: 578-606, 1973.

10. McNamara, J. A., Jr., Connelly, T. G., and McBride, M. C.: Histological studies of temporomandibular joint adaptations. In McNamara, J. A., Jr. (editor): Determinants of mandibular form and growth, Monograph No. 4, Craniofacial Growth Series, Ann Arbor, 1975, Center for Human Growth and Development, The University of Michigan, pp. 209-227.

11. McNamara, J. A., Jr., and Carlson, D. S.: Quantitative analysis of temporomandibular joint adaptations to protrusive function, AM. J. ORTHOD. 76: 593-611, 1979.

12. McNamara, J. A., Jr.: Functional determinants of craniofacial size and shape, Eur. J. Orthod. 2: 131-159, 1980.

13. Hiniker, J. J., and Ramfjord, S. P.: Anterior displacement of the mandible in adult rhesus monkeys, J. Prosthet. Dent. 16: 503512, 1966.

14. Ramfjord, S. P., and Enlow, S. P.: Anterior displacement of the 
mandible in adult rhesus monkeys: Long-term observations, J Prosthet. Dent. 26: 517-531, 1971

15. Ramfjord, S. P.. Walden, J. M., and Enlow. R. D.: Unilateral function and the temporomandibular joint in rhesus monkeys. Oral Surg. 32: 236-247, 1971.

16. Ramfjord, S. P., and Blankenship, J. R.: Increased occlusal vertical dimension in adult monkeys, J. Prosthet. Dent. 45: $74-82,1981$

17. Colico, G. L.: Le modificazoni dell' A.T.M. del Macacus rhesus a sequito di apparecchi fissi, Rass. Int. Stomatol. Prat. 9: 41-45, Suppl. 4, 1958

18. Ramfjord, S. P., and Ash, M. M., Jr.: Occlusion, Philadelphia, 1971, W. B. Saunders Company

19. Lindsay, K. N.: An autoradiographic study of cellular proliferation of the mandibular condyle after induced malocclusion in the mature rat, Arch. Oral Biol. 22: 711-714, 1978.

20. Folke, L. E. A., and Stallard, R. E.: Condylar adaptation to a change in intermaxillary relationship, J. Periodont. Res. 1: $79-89,1966$

21. Ehrlich, J., Yaffe, A., Shanfeld, J. L., Montgomery, P. C., and Davidovitch, Z : Immunochemical localization and distribution of cyclic nucleotides in the rat mandibular condyle in response to an induced occlusal change, Arch. Oral Biol. 25: 545-552, 1980.

22. Zimmermann, H. 1.: The nomal growth and remodeling of the temporomandibular joint of Macaca mulatta, M.S. thesis, University of Washington, Seattle, 1971.

23. Rushton, M. A.: Growth at the mandibular condyle in relation to some deformities, Br. Dent. J. 76: 57-68, 1944

24. Macalister, A. D.: A microscopic survey of the human temporomandibular joint, N. Z. Dent. J. 50: 161-172, 1954.

25. Thilander, B., Carlsson, G. E., and Ingervall, B.: Postnatal development of the human temporomandibular joint. I. A histologic study, Acta Odontol. Scand. 34: 117-126, 1976.

26. Tayer, B. H., and Burek, M. J.: A survey of adults' attitudes toward orthodontic therapy, AM. J. ORTHOD. 79: 305-315, 1981

27. Hurme, V. O., and van Wagenen, G.: Basic data on the emergence of permanent teeth in the rhesus monkey (Macaca mulatta), Proc. Am. Phil. Soc. 105: 105-140, 1961

28. Carlson, D. S., McNamara, J. A., Jr., Graber, L. W., and Hoffman, D. H.: Experimental studies of the temporomandibular joint. In Irby, W. B. (editor): Current concepts in oral surgery St. Louis, 1980, The C.V. Mosby Company, pp. 28-81.

29. Carlson, D. S., McNamara, J. A., Jr., and Jaul, D. H.: Histological analysis of the growth of the mandibular condyle in the rhesus monkey (Macaca mulatta), Am. J. Anat. 151: 103-118, 1978.
30. Janzen, E. K., and Bluher, A.: The cephalometric, anatomic. and histological changes in Macaca mulata after application of a continuous-acting retraction force on the mandible. AM I ORTHOD. 51: 823-855, 1965

31. Cutler, B. S., Hassig, F. N., and Turpin, D. L.: Dentofacial changes produced during and after use of a modified Milwaukee brace on Macaca mulatta. AM. J. ORTHOD. 61: 115.137, 1972.

32. Joho, J.P.: The effects of extraoral low-pull traction to the mandibular dentition of Macaca mulatta, AM. J. ORTHOD, 64: 555-577, 1973

33. Wright, D. M., and Moffett, B. C.: The postnatal development of the human temporomandibular joint, Am. J. Anat. 141: 235 250, 1974 .

34. Blackwood, H. J. J.: Growth of the mandibular condyle of the rat studied with tritiated thymidine, Arch. Oral Biol. 11: 439. 500,1966

35. Folke, L. E. A., and Stallard, R. E.: Cellular kinetics within the mandibular joint, Acta Odontol. Scand. 25: 469-489, 1967.

36. Kanouse, M. C., Ramfjord, S. P., and Nasjleti, C. E.: Condylar growth in rhesus monkeys, J. Dent. Res. 48: 1171-1176, 1969.

37. Charlier, J.-P., Petrovic, A., and Herrmann, J.: Determinisme de la croissance mandibulaire: effets de l'hyperpropulsion et de l'hormone somatotrope sur la croissance condylienne de jeunes rats, Orthod. Fr. 39: 567-579, 1968

38. Rodan, G. A., Bourret, L. A., Harvey, A., and Mensi, T.: Cyclic AMP and cyclic GMP: Mediators of mechanical effects in bone remodeling, Science 189: 467-469. 1975.

39. McNamara, J. A., Jr., and Graber, L. W.: Mandibular growth in the rhesus monkey (Macaca mulatta), Am. J. Phys. Anthropol 142: $15-24,1975$.

40. Meikle, M. C.: The effects of a Class II intermaxillary force on the dentofacial complex in the adult Macaca mulatta monkey, AM. J. ORTHOD. 58: 323-340, 1970.

41. Schneiderman, E. D., and Carlson, D. S.: Growth and remodel ing of the mandible following alteration of function in adult rhesus monkeys (Abstr.). Am. J. Phys. Anthropol. 54: 275. 1981

42. Ware, W. H., and Taylor, R. C.: Condylar repositioning following osteotomies for correction of mandibular prognathism, АM. J. ORTHOD. 54: 50-59, 1968

43. Hollender, L., and Ridell, A.: Radiography of the temporomandibular joint after oblique sliding osteotomy of the mandibular rami, Scand. J. Dent. Res. 82: 466-469, 1974.

44. Hinton, R. J., and McNamara, J. A., Jr.: Effect of age on adaptive response of adult temporomandibular joint to protrusive function in Macaca mulatta. (Submitted for publication.) 